\title{
Routing, Scheduling and Power Allocation in Generic OFDMA Wireless Networks: Optimal Design and Efficiently Computable Bounds
}

\author{
Rozita Rashtchi, Ramy H. Gohary, Senior Member, IEEE, and Halim Yanikomeroglu, Senior Member, IEEE
}

\begin{abstract}
The goal of this paper is to determine the data routes, subchannel schedules, and power allocations that maximize a weighted-sum rate of the data communicated over a generic OFDMA wireless network in which the nodes are capable of simultaneously transmitting, receiving and relaying data. Two instances are considered. In the first instance, subchannels are allowed to be time-shared by multiple links, whereas in the second instance, each subchannel is exclusively used by one of the links. Using a change of variables, the first problem is transformed into a convex form. In contrast, the second problem is not amenable to such a transformation and results in a complex mixed integer optimization problem. To develop insight into this problem, we utilize the first instance to obtain efficiently computable lower and upper bounds on the weightedsum rate that can be achieved in the absence of time-sharing. Another lower bound is obtained by enforcing the scheduling constraints through additional power constraints and a monomial approximation technique to formulate the design problem as a geometric program. Numerical investigations show that the obtained rates are higher when time-sharing is allowed, and that the lower bounds on rates in the absence of time-sharing are relatively tight.
\end{abstract}

Index Terms-Cross-layer design, geometric programming, monomial approximation, time-sharing, self-concordance.

\section{INTRODUCTION}

$\mathbf{F}$ UTURE wireless networks are expected to provide ubiquitous high data rate coverage. Meeting this expectation will bank on versatile wireless nodes that can adapt to varying channel conditions and dynamic network topologies, and that can perform multiple tasks simultaneously. For instance, for networks with both fixed and terminal relays, the nodes are expected to be capable of sending, receiving and relaying data to other nodes [1]. As such, future communication networks are expected to be less structured and more responsive to instantaneous demands. The dynamic nature of these networks give rise to a generic ad hoc topology which encompasses

Manuscript received June 10, 2013; revised September 29, 2013; accepted November 21, 2013. The associate editor coordinating the review of this paper and approving it for publication was H. Lin.

The authors are with the Department of Systems and Computer Engineering, Carleton University, Ottawa, Ontario, Canada (e-mail: \{rozita, gohary, halim\}@sce.carleton.ca).

Preliminary versions of this work were presented, in part, at the IEEE Intern. Conf. Commun. (ICC), 2012, and in part at the IEEE Glob. Telecommun. (Globecom), 2012.

This work is supported in part by BlackBerry (formerly Research In Motion (RIM)) and in part by a Collaborative Research and Development (CRD) grant of the Natural Sciences and Engineering Research Council (NSERC) of Canada.

Digital Object Identifier 10.1109/TWC.2014.012814.131045 various communication network structures, including current cellular ones and sensor networks.

To facilitate the implementation of future wireless networks, these networks are likely to rely on orthogonal frequency division multiple access (OFDMA) for accessing the wireless medium. In addition to its practicality and resilience to multiuser interference and frequency-selective fading [2], OFDMA offers an effective means for sharing the frequency band between multiple terminals, depending on their channel conditions [3]. Despite its advantages, using OFDMA in wireless networks requires relatively accurate synchronization, which can be established centrally as in the designs that will be developed hereinafter.

Another feature of future wireless networks is that the resources available for their operation are likely to be rather scarce. Such resources include the available frequency spectrum and the, typically low, power of the batteries to be used by the wireless terminals. Given the scarcity of available resources, accommodating high data rate services imposes stringent constraints on the way the communication resources are exploited. To properly exploit these resources, the routes taken by each data stream must be carefully selected, the subchannels used for transmissions must be properly scheduled, and the powers allocated to these transmissions must be judiciously determined. In particular, although the design of isolated system functionalities, e.g., routing and power allocation, simplifies the design of the communication network, these functionalities are interrelated and considering them conjointly results in more effective utilization of the available resources. A practical impediment is the computational burden incurred by the joint consideration of these functionalities. This is especially true for networks with a large number of wireless terminals with various capabilities. Hence, it is desirable to develop joint designs that are, not only close to being optimal, but also efficiently computable.

Efficient utilization of radio resources solicits the use of optimization-based techniques for designing the network. For instance, a technique based on Geometric Programming (GP) is employed in [4] to provide a suboptimal solution for power allocation in systems where the frequency band can be reused by multiple nodes. Another technique is the socalled successive polyblock approximation [5]. This technique provides the optimal power allocations for wireless networks with frequency reuse and multiple antenna nodes. For OFDMA systems with single antenna nodes, polynomialtime algorithms were developed for obtaining optimal power 
allocations in uplink [6] and downlink [7] scenarios, and for obtaining optimal binary schedules in downlink scenarios [8].

Further network performance improvements can be effected through joint consideration of multiple functionalities. For instance, jointly optimal subchannel scheduling and power allocation were obtained in [9] and [10] for OFDMA networks in which the subchannels can be time-shared among multiple nodes. When time-sharing is not allowed, an optimization problem is formulated in [11], and a heuristic inspired by the corresponding Karush-Kuhn-Tucker (KKT) conditions is developed therein to obtain jointly optimal schedules and power allocations. Elaborations on this heuristic, and analysis of its complexity and optimality aspects are provided in [12]. When the nodes are restricted to use preassigned orthogonal subchannels, the joint optimization of data routes and power allocations can be cast in an efficiently solvable convex form [13]. However, when the subchannels used by the nodes are not restricted to be orthogonal and the rates are chosen from a discrete set, a technique called nonlinear column generation is used in [14] to obtain optimal data routes and power allocations. To exploit the broadcast feature of the wireless medium, a locally optimal solution for data routes and power allocation is obtained in [15]. Therein, the nodes use superposition coding for transmission and the design is performed using the GP framework. An improvement on the design in [15] is proposed in [16] by allowing the nodes to reduce interference using a successive cancellation technique. Additional performance gains in OFDMA networks have been sought by incorporating more functionalities in the joint design of the network, e.g., [17], [18]. In [17], a heuristic algorithm is used for performing, possibly suboptimal, routing, scheduling and power allocation for networks in which each node has a prescribed role, either a source, a destination or a relay, and each subchannel is exclusively used by at most one node. A survey on recent cross-layer designs can be found in [18].

In this paper, we consider joint routing, scheduling and power allocation (JRSPA) in a generic OFDMA-based network in which each subchannel is scheduled to be used exclusively by one node at any given time instant. Such scheduling facilitates the design of the network and ensures that the transmissions of the nodes do not interfere with each other. Scenarios in which a channel is used simultaneously by multiple nodes have been considered in [19] and [20]. For the networks considered herein, the design problem is solved by a central entity that has access to the channel gains and that provides the nodes with the signalling parameters.

Two instances of networks are considered. In the first instance, the subchannels are time-shared over multiple links, as in the Long Term Evolution (LTE) systems framework [21], whereas in the second instance time-sharing is not allowed, and each subchannel is exclusively assigned to one link throughout the signalling interval, as in current cellular networks [3]. When time-sharing is allowed, the scheduling variables assume continuous values, and the design problem in this case is referred to as "continuous JRSPA". By changing the design variables, this problem is cast in a convex form that can be solved efficiently to yield the set of all the rates that can be reliably communicated over a given OFDMA-based wireless network, and the data routes, subchannel schedules and power allocations that achieve them. In contrast, when time-sharing is not allowed, the scheduling variables assume binary values, and result in a complex mixed integer optimization problem [3], which we refer to as "binary JRSPA". To alleviate the difficulty that arises in the latter case, we develop bounds on the rates that can be achieved in the binary scheduling case. To do so, we first note that, because continuous scheduling is a relaxation of binary scheduling, the weighted-sum rates it achieves constitute an upper bound on the weighted-sum rates achieved by its binary counterpart. To develop lower bounds, we observe that normalizing and rounding the (optimal) continuous schedules obtained in the case of continuous scheduling yields, potentially suboptimal, binary schedules. With these schedules fixed, the JRSPA design problem is shown to be convex and is used to obtain the first lower bound. To develop another lower bound, the restriction of the schedules to be binary is captured by imposing a set of constraints on the power allocations. Although the resulting formulation is non-convex, by manipulating it, the JRSPA design problem is cast in a form that is amenable to an efficient GP-based approximation technique [22]. This technique yields locally optimal routes and power allocations, which are subsequently used to recover the binary schedules. Numerical results show that networks that utilize time-sharing can achieve significantly higher rates than networks in which time-sharing is not allowed, and that the advantage of timesharing becomes more pronounced as the network size increases. When time-sharing is not allowed, numerical results suggest that the gap between the lower bounds and the true maximum is usually small, specially at low signal-to-noiseratios (SNRs).

In comparison with [9], in this paper we incorporate routing jointly with scheduling and power allocation in the design framework, and provide a GP-based lower bound for the case with no time-sharing, which is not considered in [9]. In comparison with [4], we consider joint routing, scheduling and power allocations, whereas in [4] only power allocation is considered. In particular, in [4] no relaying or any of the associated routing and scheduling constraints are considered. Furthermore, in this paper we provide a complexity analysis for all considered schemes and also equivalent self-concordant formulations to bound the number of Newton iterations required for solving the optimization problems that underlie our cross-layer designs.

The paper is organized as follows. In Section II, we provide the network flow and communication models. In Section III, we provide mathematical characterization of the constraints that should be satisfied by the design variables, and subsequently a preliminary version of the JRSPA design problem is formulated. The case of continuous scheduling is considered in Section IV, and a convex formulation that yields the jointly optimal data routes, subchannel schedules and power allocations is provided. The case of binary scheduling is considered in Section V, and formulations for obtaining lower bounds on the weighted-sum rates that can be achieved in this case are developed. The computational complexity of the proposed designs are examined in Section VI. Section VII provides numerical examples and Section VIII concludes the paper. GP definitions, analyses pertaining thereto and equivalent self- 


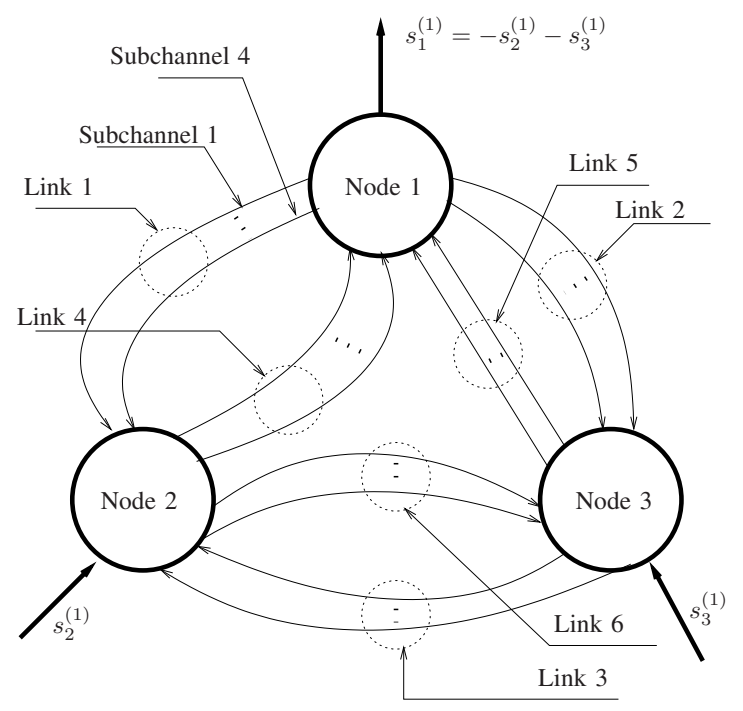

Fig. 1: Network schematic with $N=3, L=6, K=4$ and $D=1$.

concordant formulations are provided in the appendices.

\section{SySTEM MOdEL}

We consider a generic OFDMA-based wireless network of $N$ nodes and $L$ directed links. Each node is assumed to have one transmit and one receive antenna, and a power budget, $P_{n}, n \in \mathcal{N} \triangleq\{1,2, \cdots, N\}$. In addition, each node is assumed to be capable of simultaneously transmitting, receiving and relaying data to other nodes using the decodeand-forward scheme. It will be shown hereinafter that this assumption encompasses cases in which some nodes are constrained to performing a subset of these tasks. For practical considerations, the relaying nodes are assumed to operate in the half duplex mode, whereby each node uses distinct physical channels for transmission and reception.

The available OFDMA frequency spectrum, $W_{0}$, is divided into $K$ narrowband subchannels, each of bandwidth $W=\frac{W_{0}}{K}$. The $K$ subchannels are assumed to remain essentially constant during the entire signalling interval. The set of all destination nodes is denoted by $\mathcal{D} \triangleq\{1,2, \cdots, D\}$, and the set of all links is denoted by $\mathcal{L} \triangleq\{1, \ldots, L\}$, where $L=N(N-1)$. The sets of incoming and outgoing links of node $n \in \mathcal{N}$ are denoted by $\mathcal{I}(n)$ and $\mathcal{O}(n)$, respectively. Each link $\ell \in \mathcal{L}$ is composed of $K$ subchannels, each with a complex coefficient, $h_{\ell k}, k \in \mathcal{K} \triangleq\{1 \cdots, K\}$. This network can be represented by a directed graph with $N$ vertices and $L K$ edges, which are weighted by the subchannel gains, $\left\{\left|h_{\ell k}\right|^{2}\right\}$. An instance of such a network with $N=3$ nodes, $L=6$ directed links and $K=4$ subchannels is depicted in Figure 1. The connectivity of the graph can be characterized by the incidence matrix, $A=\left[a_{n \ell}\right]$, where $a_{n \ell}=1$ if $\ell \in \mathcal{O}(n), a_{n \ell}=-1$ if $\ell \in \mathcal{I}(n)$ and $a_{n \ell}=0$ otherwise [13].

To maximize the network utility, the routes taken by each data stream, the schedules needed for assigning subchannels to links, and the power and the rates assigned to each transmission must be judiciously determined. These decisions have traditionally been considered separately albeit significantly higher gains can be achieved when these decisions are made jointly. This task will be the focus of the following sections: In Section III we will provide an optimization framework for the joint design and in Sections IV and V this framework will be used to obtain bounds on the maximum weighted-sum rate that can be supported by the network.

\section{The Optimization Framework}

In this section we will provide the design objective and a characterization of the constraints that must be satisfied by feasible data routes, subchannel schedules and power allocations.

\section{A. System Objective}

Let $s_{n}^{(d)}$ be the rate of the data stream injected into node $n \in \mathcal{N}$ and intended for destination $d \in \mathcal{D}$, and let $w_{n}^{(d)}$ be the non-negative weight assigned to $s_{n}^{(d)}$. Our objective is to maximize the weighted-sum of the rates injected into the network, i.e., $\max \sum_{d \in \mathcal{D}} \sum_{n \in \mathcal{N}, n \neq d} w_{n}^{(d)} s_{n}^{(d)}$, for some given $\left\{w_{n}^{(d)}\right\}$ satisfying $\frac{1}{D(N-1)} \sum_{d \in \mathcal{D}} \sum_{n \in \mathcal{N} \backslash\{d\}} w_{n}^{(d)}=1$. A particular weight assignment is when all rates are assigned the same weight, i.e., $w_{n}^{(d)}=1$, for all $n \in \mathcal{N}$ and $d \in \mathcal{D}$.

From a practical perspective, assigning weights to injected rates provides a convenient means for controlling the quality of service (QoS) offered to various network destinations; a higher weight implies a higher priority to the corresponding rate. Such weights are typically assigned a priori, but can be adapted to meet variations in the QoS requirements [10]. Another advantage of considering weighted-sum rates is that varying the weights over the unit simplex enables us to evaluate the region of all injected rates that can be communicated over the network during a signalling interval. To see the utility of assigning weights to rates rather than destinations, we note that, since there are multiple sources, a destination may wish to assign different priorities to different sources. Hence, assigning weights to rates is more general than assigning them to destinations.

\section{B. System Constraints}

To ensure realizability of the prospective design, data routes must satisfy network layer constraints, whereas subchannel schedules and power allocations must satisfy medium access control (MAC) layer constraints. The constraints from both network and MAC layers are coupled by the capacity of each wireless link, which imposes a physical layer constraint. These constraints, their implications, and their interdependence will be elucidated in this section.

1) Routing Constraints: Characterizing the data routes between various source-destination pairs can be effected through the data flows on all subchannels of each link. The flows are distinguished by the intended destination. In particular, let $x_{\ell k}^{(d)}$ be the data flow intended for destination $d \in \mathcal{D}$ on subchannel $k \in \mathcal{K}$ of link $\ell \in \mathcal{L}$. The flows, $\left\{x_{\ell k}^{(d)}\right\}$, and the injected rates, $\left\{s_{n}^{(d)}\right\}$, are related by the flow conservation law, which must be satisfied at each node in the network. This law stipulates that the sum of all flows intended for each destination $d \in \mathcal{D}$ at each node must be equal to zero [13]. This guarantees the existence of continuous routes 
between sources and destinations. Hence, to obtain the routes that enable the maximum weighted-sum rate to be achieved, we will include $\left\{x_{\ell k}^{(d)}\right\}$ in the cross-layer design framework. Using the incidence matrix defined in Section II to distinguish between incoming and outgoing links, it can be seen that $\left\{x_{\ell k}^{(d)}\right\}$ and $\left\{s_{n}^{(d)}\right\}$ must satisfy the following constraints:

$$
\sum_{\ell \in \mathcal{L}} \sum_{k \in \mathcal{K}} a_{n l} x_{\ell k}^{(d)}=s_{n}^{(d)}, \quad \forall n \in \mathcal{N}, \forall d \in \mathcal{D}, n \neq d .
$$

Successive application of the flow conservation law yields that the total data rate received by any destination node $d \in \mathcal{D}$ is given by $s_{d}^{(d)}=-\sum_{n \in \mathcal{N} \backslash\{d\}} s_{n}^{(d)}$, where $\backslash$ is the set minus operation. The fact that $s_{d}^{(d)}$ is a negative quantity implies that this rate "leaves" the network.

Injected rates, $\left\{s_{n}^{(d)}\right\}_{n \neq d}$, and data flows, $\left\{x_{\ell k}^{(d)}\right\}$, must be non-negative. Hence,

$$
\begin{array}{ll}
x_{\ell k}^{(d)} \geq 0, & \forall \ell \in \mathcal{L}, \forall k \in \mathcal{K}, \forall d \in \mathcal{D}, \\
s_{n}^{(d)} \geq 0, & \forall n \in \mathcal{N}, \quad \forall d \in \mathcal{D}, n \neq d .
\end{array}
$$

For mathematical tractability, the source nodes will be assumed to have an infinite backlog, and thus they always have data ready for transmission [18].

2) Scheduling Constraints: To characterize the constraints that must be satisfied by subchannel schedules, let $c_{\ell k}$ be a variable that determines the fraction of time during which link $\ell \in \mathcal{L}$ is scheduled to use subchannel $k \in \mathcal{K}$. When timesharing is allowed, $\left\{c_{\ell k}\right\}$ assume continuous values, whereas when time-sharing is not allowed, $\left\{c_{\ell k}\right\}$ assume binary values. Hence, with time-sharing,

$$
c_{\ell k} \in[0,1], \quad \forall \ell \in \mathcal{L}, \forall k \in \mathcal{K},
$$

and without time-sharing,

$$
c_{\ell k} \in\{0,1\}, \quad \forall \ell \in \mathcal{L}, \forall k \in \mathcal{K} .
$$

In the considered OFDMA structure, interference is avoided by restricting each subchannel to be used at most once across the entire network [23]. Hence, with (4) or (5) satisfied, depending on whether time-sharing is allowed or not, this requirement can be expressed as

$$
\sum_{\ell \in \mathcal{L}} c_{\ell k} \leq 1, \quad \forall k \in \mathcal{K} .
$$

3) Power Allocation Constraints: To determine the constraints that must be satisfied by any feasible power allocation, let $p_{\ell k}$ denote the power allocated to subchannel $k \in \mathcal{K}$ of link $\ell \in \mathcal{L}$. The elements of $\left\{p_{\ell k}\right\}$ must satisfy

$$
p_{\ell k} \geq 0, \quad \forall \ell \in \mathcal{L}, \forall k \in \mathcal{K} .
$$

In a practical network, each node $n \in \mathcal{N}$ has a power budget, $P_{n}$, which bounds the total power allocated by each node on outgoing links and subchannels. This constraint can be written as

$$
\sum_{k \in \mathcal{K}} \sum_{\ell \in O(n)} c_{\ell k} p_{\ell k} \leq P_{n}, \quad \forall n \in \mathcal{N} .
$$

In writing (8), we have used the fact that only the subchannels scheduled to outgoing links contribute to the power consumed by every node.
4) Capacity Constraints: The data routes, subchannel schedules and power allocations are coupled by the maximum rate that can be reliably communicated on the subchannels of each link, i.e., subchannel capacities. In other words, the aggregate flow on each subchannel $k \in \mathcal{K}$, of each link $\ell \in \mathcal{L}$, $\sum_{d=1}^{D} x_{\ell k}^{(d)}$, must not exceed the capacity of this subchannel. Assuming that the nodes use Gaussian signaling, the capacity of subchannel $k \in \mathcal{K}$ of link $\ell \in \mathcal{L}$ can be expressed as $W \log _{2}\left(1+\frac{p_{\ell k}\left|h_{\ell k}\right|^{2}}{W N_{0}}\right)$, where $N_{0}$ is the spectral density of the additive white Gaussian noise at destination nodes. When the $k$-th subchannel is used on the $\ell$-th link for a fraction $c_{\ell k}$ of the signalling interval, the aggregate flow on this subchannel must not exceed $W c_{\ell k} \log _{2}\left(1+\frac{p_{\ell k}\left|h_{\ell k}\right|^{2}}{W N_{0}}\right)$, that is,

$$
\sum_{d \in \mathcal{D}} x_{\ell k}^{(d)} \leq W c_{\ell k} \log _{2}\left(1+\frac{p_{\ell k}\left|h_{\ell k}\right|^{2}}{W N_{0}}\right), \forall \ell \in \mathcal{L}, \forall k \in \mathcal{K}
$$

The following remark exposes the generality of the considered model.

\section{Remark 1 (Special Configurations).}

- Prohibiting a node from being a source can be accomplished by setting its injected rate to zero, and prohibiting a node from being a destination can be accomplished by excluding its index from $\mathcal{D}$.

- Restricting a node to be a source can be accomplished by setting all the subchannel gains of incoming links to zero. Likewise, restricting a node to be a destination can be accomplished by setting all the subchannel gains of outgoing links to zero.

\section{The Case of Continuous JRSPA: Optimal Design}

In this section we consider the case when subchannels can be time-shared among multiple links. This case corresponds to the situation in which $\left\{c_{\ell k}\right\}$ satisfy the constraints in (4). Combining the objective in Section III-A with the the constraints in (1)-(4) and (6)-(9), the JRSPA design problem can be cast as the following optimization problem:

$$
\begin{array}{ll}
\max _{\left\{s_{n}^{(d)}\right\},\left\{x_{\ell k}^{(d)}\right\},\left\{c_{\ell k}\right\},\left\{p_{\ell k}\right\}} & \sum_{d} \sum_{n, n \neq d} w_{n}^{(d)} s_{n}^{(d)} \\
\text { subject to } & \\
\sum_{\ell \in \mathcal{L}} \sum_{k \in \mathcal{K}} a_{n \ell} x_{\ell k}^{(d)}=s_{n}^{(d)}, & \forall n \in \mathcal{N}, \forall d \in \mathcal{D}, n \neq d, \\
x_{\ell k}^{(d)} \geq 0, & \forall \ell \in \mathcal{L}, \forall k \in \mathcal{K}, \forall d \in \mathcal{D}, \\
s_{n}^{(d)} \geq 0, & \forall n, \forall d \in \mathcal{D}, n \neq d, \\
\sum_{d \in \mathcal{D}} x_{\ell k}^{(d)} \leq W c_{\ell k} \log _{2}\left(1+\frac{p_{\ell k}\left|h_{\ell k}\right|^{2}}{W N_{0}}\right), & \forall \mathcal{L}, \forall k \in \mathcal{K}, \\
c_{\ell k} \in[0,1], & \forall \ell \in \mathcal{L}, \forall k \in \mathcal{K}, \forall k \in \mathcal{K}, \\
p_{\ell k} \geq 0, & \forall k \in \mathcal{K}, \\
\sum_{\ell \in \mathcal{L}} c_{\ell k} \leq 1, & \forall n \in \mathcal{N} . \\
\sum_{k \in \mathcal{K}} \sum_{\ell \in O(n)} c_{\ell k} p_{\ell k} \leq P_{n}, &
\end{array}
$$


The objective and the constraints in this formulation are linear, except the constraints in (10e) and (10i). For the constraint in (10e), the left hand side (LHS) is linear. However, as can be readily verified by direct computation of the Hessian matrix, the right hand side (RHS) of (10e) is not jointly concave in $\left(c_{\ell k}, p_{\ell k}\right)$. For the constraint in (10i), the RHS is linear, but the LHS is not jointly convex in $c_{\ell k}$ and $p_{\ell k}$, as can be verified by direct computation of the Hessian matrix.

The non-convexity of (10e) and (10i) renders the JRSPA design in (10) difficult to solve. This difficulty can be circumvented by using the following change of variables. Let

$$
y_{\ell k}=c_{\ell k} p_{\ell k}, \quad \forall \ell \in \mathcal{L}, \forall k \in \mathcal{K} .
$$

Since $\left\{c_{\ell k}\right\}$ are nonnegative, it can be seen that this change of variables yields a one-to-one mapping from $\left\{y_{\ell k}\right\}$ to $\left\{p_{\ell k}\right\}$; when $c_{\ell k}=0, p_{\ell k}$ can be set to zero without loss of optimality. A similar change of variables was used in [9] in a different optimization framework. Using $\left\{y_{\ell k}\right\}$ instead of $\left\{p_{\ell k}\right\}$, the RHS of (10e) can be expressed as $W c_{\ell k} \log _{2}\left(1+\frac{y_{\ell k}\left|h_{\ell k}\right|^{2}}{c_{\ell k} W N_{0}}\right)$.

We now recall the following result from [24]. Let $g(u)$ be a function and $f(t, u)$ be its perspective: $f(t, u) \triangleq t g\left(\frac{u}{t}\right)$. Then $g(u)$ is concave in $u$ if and only if $f(t, u)$ is concave in $(t, u)$. Now, $W c_{\ell k} \log _{2}\left(1+\frac{y_{\ell k}\left|h_{\ell k}\right|^{2}}{c_{\ell k} W N_{0}}\right)$ is the perspective of $W \log _{2}\left(1+\frac{y_{\ell k}\left|h_{\ell k}\right|^{2}}{W N_{0}}\right)$. Since this function is concave in $y_{\ell k}$, we conclude that $W c_{\ell k} \log _{2}\left(1+\frac{y_{\ell k}\left|h_{\ell k}\right|^{2}}{c_{\ell k} W N_{0}}\right)$ is concave in $\left(c_{\ell k}, y_{\ell k}\right)$.

Using this observation and the change of variables in (11), the JRSPA problem can be cast in the following convex form:

$$
\begin{array}{ll}
\max _{\left\{s_{n}^{(d)}\right\},\left\{x_{\ell k}^{(d)}\right\},\left\{c_{\ell k}\right\},\left\{y_{\ell k}\right\}} \sum_{d} \sum_{n, n \neq d} w_{n}^{(d)} s_{n}^{(d)} \\
\text { subject to } \\
\sum_{\ell \in \mathcal{L}} \sum_{k \in \mathcal{K}} a_{n \ell} x_{\ell k}^{(d)}=s_{n}^{(d)}, & \forall n \in \mathcal{N}, \forall d \in \mathcal{D}, n \neq d, \\
x_{\ell k}^{(d)} \geq 0, & \forall \ell \in \mathcal{L}, \forall k \in \mathcal{K}, \forall d \in \mathcal{D}, \\
s_{n}^{(d)} \geq 0, & \forall n \in \mathcal{N}, \forall d \in \mathcal{D}, n \neq d, \\
\sum_{d \in \mathcal{D}} x_{\ell k}^{(d)} \leq W c_{\ell k} \log _{2}\left(1+\frac{y_{\ell k}\left|h_{\ell k}\right|^{2}}{c_{\ell k} W N_{0}}\right), & \forall \ell \in \mathcal{L}, \forall k \in \mathcal{K}, \\
c_{\ell k} \geq 0, y_{\ell k} \geq 0, & \forall \ell \in \mathcal{L}, \forall k \in \mathcal{K}, \\
\sum_{\ell \in \mathcal{L}} c_{\ell k} \leq 1, & \forall k \in \mathcal{K}, \\
\sum_{k \in \mathcal{K}} \sum_{\ell \in \mathcal{O}(n)} y_{\ell k} \leq P_{n}, & \forall n \in \mathcal{N} .
\end{array}
$$

From this formulation it can be seen that the objective is linear and all, but (12e), are linear constraints. However, we have shown that (12e) is also convex. Hence, we conclude that (12) is a convex optimization problem which can be solved efficiently. Being strictly feasible, it can be seen that the global optimal solution of this problem can be found in polynomial time [24].

\section{The CASE OF BINARY JRSPA: EFFICIENTLY COMPUTABLE BOUNDS}

The difference between continuous and binary JRSPA is induced by the scheduling constraints: In continuous JRSPA, the scheduling variables can take on any value in the interval $[0,1]$, whereas in the binary JRSPA, these variables can be either 0 or 1 . This difference renders solutions of the continuous JRSPA not necessarily feasible for the binary one. Having considered the continuous case in Section IV, in this section we consider the binary case, i.e., the case in which time-sharing is not allowed. In this case the JRSPA problem in (10) will be reformulated as

$$
\begin{aligned}
& \max _{\left\{s_{n}^{(d)}\right\},\left\{x_{\ell k}^{(d)}\right\},\left\{c_{\ell k}\right\},\left\{p_{\ell k}\right\}} \sum_{d} \sum_{n, n \neq d} w_{n}^{(d)} s_{n}^{(d)}, \\
& \text { subject to } \quad(10 \mathrm{~b})-(10 \mathrm{e}) \text { and }(10 \mathrm{~g})-(10 \mathrm{i}), \\
& \\
& c_{\ell k} \in\{0,1\}, \quad \forall \ell \in \mathcal{L}, \forall k \in \mathcal{K} .
\end{aligned}
$$

This problem is in the form of mixed integer non-linear programming and, because of the binary constraints in (13b), can be shown to be computationally prohibitive, even for relatively small networks. To avoid solving (13) directly, we will develop efficiently computable, and relatively tight, bounds on the weighted-sum rate that it yields.

Before developing these bounds, we note that, for any given set of subchannel schedules, $\left\{c_{\ell k}\right\}$, the optimization problem in (13) is convex. Hence, its global optimal can be obtained by solving it for all possible choices of $\left\{c_{\ell k}\right\}$ and choosing the set that yields the maximum weighted-sum rate. The complexity of this approach is exponential in $K L$, cf. Section VI.

An upper bound on the weighted-sum rate yielded by (13) can be readily obtained by noting that the formulation in (12) corresponds to a relaxation of the formulation in (13). Since the formulation in (12) is convex, it can be seen that, for a given set of weights, $\left\{w_{n}^{(d)}\right\}$, it yields a rate vector that dominates the one yielded by (13) for the same set of weights. In other words, (12) yields an outer bound on the set of rates achieved by (13).

A lower bound on the weighted-sum rate yielded by (13) can obtained by fixing any feasible set of binary of binary schedules, $\left\{c_{\ell k}\right\}$, that satisfy $(12 \mathrm{~g})$ and solving the resulting convex problem. One way to select the binary schedules is by normalizing and rounding the continuous schedules generated by the convex formulation in (12).

Another lower bound on the weighted-sum rate yielded by (13) can obtained by inducing the effect of binary schedules through a set of constraints on the power allocations. This approach results in a new formulation that, despite being nonconvex, it shares many of the features of the GP-standard form, cf. Appendix A-1, and is amenable to the monomial approximation technique in Appendix A-2. This approach and the one based on rounding will be described next.

\section{A. The Rounding-Based Approach}

In this approach, we consider the continuous subchannel schedules, $\hat{c}_{\ell k} \in[0,1], \forall \ell \in \mathcal{L}, \forall k \in \mathcal{K}$, obtained from the convex formulation in (12). To construct a set of, potentially suboptimal, binary schedules, $\left\{\tilde{c}_{\ell k}\right\}$ from continuous ones, $\left\{\hat{c}_{\ell k}\right\}$, for every $k \in \mathcal{K}$, we set the element of $\left\{\tilde{c}_{\ell k}\right\}_{\ell=1}^{L}$ corresponding to the largest element of $\left\{\hat{c}_{\ell k}\right\}_{\ell=1}^{L}$ to 1 and 
the other elements to 0 ; i.e.,

$$
\tilde{c}_{\ell k}=\left\lfloor\frac{\hat{c}_{\ell k}}{\max _{\ell^{\prime} \in \mathcal{L}} \hat{c}_{\ell^{\prime} k}}\right\rfloor, \quad \forall \ell \in \mathcal{L}, \forall k \in \mathcal{K} .
$$

By construction, the elements of $\left\{\tilde{c}_{\ell k}\right\}$ satisfy the scheduling feasibility constraints in (13), i.e., (10h) and (13b), and can hence be used as if they were the optimal subchannel schedules. With $\left\{\tilde{c}_{\ell k}\right\}$ fixed, the JRSPA problem in (13) can be cast in the following convex form:

$$
\begin{array}{ll}
\max _{\left\{s_{n}^{(d)}\right\},\left\{x_{\ell k}^{(d)}\right\},\left\{p_{\ell k}\right\}} & \sum_{d \in \mathcal{D}} \sum_{n \in \mathcal{N}, n \neq d} w_{n}^{(d)} s_{n}^{(d)}, \\
\text { subject to } & \\
\sum_{\ell \in \mathcal{L}} \sum_{k \in \mathcal{K}} a_{n \ell} x_{\ell k}^{(d)}=s_{n}^{(d)}, & \forall n \in \mathcal{N}, \forall d \in \mathcal{D}, n \neq d, \\
x_{\ell k}^{(d)} \geq 0, & \forall \ell \in \mathcal{L}, \forall k \in \mathcal{K}, \forall d \in \mathcal{D}, \\
s_{n}^{(d)} \geq 0, & \forall n \in \mathcal{N}, \forall d \in \mathcal{D}, n \neq d, \\
\sum_{d \in \mathcal{D}} x_{\ell k}^{(d)} \leq W \tilde{c}_{\ell k} \log _{2}\left(1+\frac{p_{\ell k}\left|h_{\ell k}\right|^{2}}{W N_{0}}\right), & \forall \ell \in \mathcal{L}, \forall k \in \mathcal{K}, \\
p_{\ell k} \geq 0, & \forall n \in \mathcal{N}, \forall k \in \mathcal{K}, \\
\sum_{k \in \mathcal{K}} \sum_{\ell \in \mathcal{O}(n)} \tilde{c}_{\ell k} p_{\ell k} \leq P_{n}, & \forall n \in \mathcal{N} .
\end{array}
$$

Since the schedules $\left\{\tilde{c}_{\ell k}\right\}$ are fixed, the formulation in (15) can be seen to be convex. Since this formulation is based on potentially suboptimal schedules, the weighted-sum rate it generates is a lower bound on the corresponding sum rate generated by the problem with binary schedules.

\section{B. The GP-Based Approach}

We now consider the other lower bound on the weightedsum rate achieved by the formulation in (13). We begin by noting that the scheduling constraints in (13b) and (10h) imply that subchannel $k \in \mathcal{K}$ can be used on at most one link. From (9), it can be seen that, when $c_{\ell k}$ is equal to zero, setting $p_{\ell k}=0$ does not incur loss of optimality. Hence, the constraints in (13b) and (10h) can be enforced by allowing at most one link to have a strictly positive power on any subchannel $k \in \mathcal{K}$. In other words, the binary scheduling constraints are equivalent to

$$
p_{\ell k} p_{\ell^{\prime} k}=0, \quad \forall k \in \mathcal{K}, \forall \ell, \ell^{\prime} \in \mathcal{L}, \ell \neq \ell^{\prime} .
$$

Enforcing this constraint yields to at most one 'active' link per subchannel, which makes the recovery of the binary schedules from the power allocations straightforward.

The replacement of the constraints in (13b) and (10h) with the ones in (16) does not affect the flow conservation in (10b) and the non-negativity constraints in (10c), (10d) and (10g). However, this replacement affects the capacity constraints in (10e) and the power budget constraints in (10i). Since, from (16), only one element in $\left\{p_{\ell k}\right\}_{\ell=1}^{L}$ is strictly positive for any subchannel $k \in \mathcal{K}$, the constraints in (10e) can be expressed as

$$
\sum_{d \in \mathcal{D}} x_{\ell k}^{(d)} \leq W \log _{2}\left(1+\frac{p_{\ell k}\left|h_{\ell k}\right|^{2}}{W N_{0}}\right), \quad \forall \ell \in \mathcal{L}, \forall k \in \mathcal{K},
$$

and the constraint in (10i) can be expressed as

$$
\sum_{k \in \mathcal{K}} \sum_{\ell \in \mathcal{O}(n)} p_{\ell k} \leq P_{n}, \quad \forall n \in \mathcal{N} .
$$

Replacing the constraints in (10e), (13b), (10h) and (10i) with those in (16)-(18) yields an analogous formulation in which the binary schedules are accounted for by equivalent constraints on the power allocations of the links. This formulation can be expressed as

$$
\begin{aligned}
& \max _{\left\{s_{n}^{(d)}\right\},\left\{x_{\ell k}^{(d)}\right\},\left\{p_{\ell k}\right\}} \sum_{d \in \mathcal{D}} \sum_{n \in \mathcal{N}, n \neq d} w_{n}^{(d)} s_{n}^{(d)}, \\
& \text { subject to } \quad(10 \mathrm{~b})-(10 \mathrm{~d}), \\
& \sum_{d \in \mathcal{D}} x_{\ell k}^{(d)} \leq W \log _{2}\left(1+\frac{p_{\ell k}\left|h_{\ell k}\right|^{2}}{W N_{0}}\right), \\
& \sum_{k \in \mathcal{K}} \sum_{\ell \in \mathcal{O}(n)} p_{\ell k} \leq P_{n}, \quad \forall n \in \mathcal{N}, \\
& P_{\ell k} \geq 0, \quad \forall \ell \in \mathcal{L}, \forall k \in \mathcal{K}, \\
& p_{\ell k} p_{\ell^{\prime} k}=0, \quad \forall \ell \in \mathcal{L}, \forall k \in \mathcal{K},
\end{aligned}
$$

The optimization problem in (19) is nonconvex and therefore difficult to solve. However, this problem would have been easier to solve had it been possible to ignore the ' 1 ' in the argument of the $\log (\cdot)$ function in (19c), for instance when $\frac{p_{\ell k}\left|h_{\ell k}\right|^{2}}{W N_{0}}$ is sufficiently large [4]. Unfortunately, such an approximation is not possible in the current context because the constraints in (19f) imply that, while one link operates at high SNR, the others must operate at zero SNRs. Indeed, one of the tasks underlying the formulation in (19) is to determine which link powers ought to be set to zero, and since this information is not available a priori one cannot use the highSNR approximation methodology in [4].

The optimization problem in (19), although nonconvex, is amenable to GP-based monomial approximation. In order to use this approximation, we use the exponential function to map $\left\{s_{n}^{(d)}\right\}$ and $\left\{x_{\ell k}^{(d)}\right\}$ to $\left\{t_{n}^{(d)}\right\}$ and $\left\{r_{\ell k}^{(d)}\right\}$, respectively. In particular, we have

$$
\begin{aligned}
& s_{n}^{(d)}=\log _{2} t_{n}^{(d)}, \quad \forall n \in \mathcal{N}, \forall d \in \mathcal{D}, n \neq d, \\
& x_{\ell k}^{(d)}=W \log _{2} r_{\ell k}^{(d)}, \quad \forall \ell \in \mathcal{L}, \forall k \in \mathcal{K}, \forall d \in \mathcal{D} .
\end{aligned}
$$

These are one-to-one mappings which enable the original variables to be readily recovered from the new ones. Using the new variables, the objective and the constraints in (19a)(19b) can be readily expressed in GP compatible forms; cf. Appendix A-1. For the constraints in (19f), although the LHSs are in the form of monomials, they are not compatible with the GP framework because their RHSs are not equal to 1 . This difficulty can be readily alleviated by replacing (16) with constraints of the form $p_{\ell k} p_{\ell^{\prime} k} \leq \epsilon$, where $\epsilon$ is a small positive number. This replacement will expand the feasible region of the considered optimization problem in (19). Now, using the new variables, the capacity constraints in (19c) can be expressed as

$$
W N_{0} \prod_{d \in \mathcal{D}} r_{\ell k}^{(d)} \leq W N_{0}+p_{\ell k}\left|h_{\ell k}\right|^{2}, \quad \forall \ell \in \mathcal{L}, \forall k \in \mathcal{K} .
$$


The RHS of each constraint in (22) is not a monomial and hence, does not conform to the GP framework. To overcome this difficulty, we invoke the monomial approximation method described in Appendix A-2. Using this method, the expression in (29) is used to approximate the RHSs of (22) with monomial functions near some initial power allocation, $\left\{p_{\ell k}^{(0)}\right\}$.

We now consider the remaining constraints. The nonnegativity constraints on the allocated powers in (19e) are inherently incorporated in the GP framework. Finally, the constraints in (19d) are compatible with the GP standard form.

Now, assuming that a feasible initial power allocation, $\left\{p_{\ell k}^{(0)}\right\}$, is given, the maximum weighted-sum rate around $\left\{p_{\ell k}^{(0)}\right\}$ can be approximated by solving the following GP:

$$
\max _{\left\{t_{n}^{(d)}\right\},\left\{r_{\ell k}^{(d)}\right\},\left\{p_{n}^{(k)}\right\}} \prod_{d \in \mathcal{D}} \prod_{n \in \mathcal{N}, n \neq d}\left(t_{n}^{(d)}\right)^{w_{n}^{(d)}},
$$

subject to

$$
\begin{array}{ll}
\prod_{\ell \in \mathcal{L}} \prod_{k \in \mathcal{K}}\left(r_{\ell k}^{(d)}\right)^{W a_{n \ell}}=t_{n}^{(d)}, & \forall n \in \mathcal{N}, \forall d \in \mathcal{D}, n \neq d, \\
t_{n}^{(d)} \geq 1, & \forall n \in \mathcal{N}, \forall d \in \mathcal{D}, n \neq d, \\
r_{\ell k}^{(d)} \geq 1, & \forall \ell \in \mathcal{L}, \forall k \in \mathcal{K}, \forall d \in \mathcal{D}, \\
W N_{0} \prod_{d \in \mathcal{D}} r_{\ell k}^{(d)} \leq q_{\ell k}\left(p_{\ell k} / p_{\ell k}^{(0)}\right)^{\theta_{\ell k}}, \quad \forall k \in \mathcal{K}, \forall \ell \in \mathcal{L}, & \forall n \in \mathcal{N}, \\
\sum_{k \in \mathcal{K}} \sum_{\ell \in \mathcal{O}(n)} p_{\ell k} \leq P_{n}, & \forall k \in \mathcal{K}, \forall \ell, \ell^{\prime} \in \mathcal{L}, \ell \neq \ell^{\prime}, \\
p_{\ell k} p_{\ell^{\prime} k} \leq \epsilon, & \forall
\end{array}
$$

where $q_{\ell k}=W N_{0}+p_{0, n}^{(k)}\left|h_{n n^{\prime}}^{(k)}\right|^{2}$, and $\theta_{\ell k}=\frac{p_{0, n}^{(k)}\left|h_{n n^{\prime}}^{(k)}\right|^{2}}{q_{\ell k}}$. Using a standard logarithmic transformation, (23) can be readily converted to an efficiently solvable convex form [22].

1) Iterative Monomial Approximation: Finding the global solution for the nonconvex problem in (19) is difficult, whereas solving the approximated problem in (23) is straightforward. To exploit this fact, we incorporate the formulation in (23) in an iterative algorithm. In particular, starting from a feasible $\left\{p_{\ell k}^{(0)}\right\}$, the problem in (23) is transformed into a convex form and solved using an interior point solver. The output power allocation resulting from solving this problem is then used as the initial power allocation in the following iteration. This technique is referred to as the single condensation method and, under relatively mild conditions, is guaranteed to converge to a solution that satisfies the KKT conditions corresponding to (19) with (19f) replaced with (23h), cf. Appendix B. Since the problem in (19) with (19f) replaced with (23h) is not convex, the KKT conditions are only necessary for optimality and the resulting solution is a lower bound on the achievable weighted-sum rate on the expanded feasible region. This implies that, if the initial point for the iterative monomial approximation technique happens to be appropriately chosen, the GP approach would yield the optimal design. Unfortunately, finding such an initial point depends on the network structure and is generally elusive.

2) Obtaining a Feasible Solution: The solution yielded by the relaxation in $(23 \mathrm{~h})$ is projected onto the feasible region of the original problem in (19). In particular, let $\left\{\hat{p}_{\ell k}\right\}$ be the power allocation attained by the iterative monomial approximation in Section V-B1. Then, the projection of $\left\{\hat{p}_{\ell k}\right\}$ onto the feasible region of (19) can expressed as

$$
\tilde{p}_{\ell k}=\left\lfloor\frac{\hat{p}_{\ell k}}{\max _{\ell^{\prime} \in \mathcal{L}} \hat{p}_{\ell^{\prime} k}}\right\rfloor \max _{\ell^{\prime} \in \mathcal{L}} \hat{p}_{\ell^{\prime} k}, \quad \forall \ell \in \mathcal{L}, \forall k \in \mathcal{K} .
$$

We now use $\left\{\tilde{p}_{\ell k}\right\}$, as if they were the optimal power allocations, to retrieve the subchannel schedules. In particular, from (16), it can be seen that, $c_{\ell k}=0$ when $p_{\ell k}=0$ and $c_{\ell k}=1$ when $p_{\ell k}>0$. Now, with $\left\{\tilde{p}_{\ell k}\right\}$ fixed, the optimal routes can be obtained by solving (19) for $\left\{x_{\ell k}^{(d)}\right\}$. In particular, $\left\{x_{\ell k}^{(d)}\right\}$ can be obtained by solving the following linear program:

$$
\max _{\left\{s_{n}^{(d)}\right\},\left\{x_{\ell k}^{(d)}\right\}} \sum_{d \in \mathcal{D}} \sum_{n \in \mathcal{N} \backslash\{d\}} w_{n}^{(d)} s_{n}^{(d)},
$$

subject to

$$
\begin{gathered}
s_{n}^{(d)} \geq 0, \\
\sum_{\ell \in \mathcal{L}} \sum_{k \in \mathcal{K}} a_{n \ell} x_{\ell k}^{(d)}=s_{n}^{(d)}, \quad \forall n \in \mathcal{N}, \forall d \in \mathcal{D}, \quad n \neq d, \\
x_{\ell k}^{(d)} \geq 0, \quad \forall d \in \mathcal{D}, n \neq d, \\
\sum_{d \in \mathcal{D}} x_{\ell k}^{(d)} \leq W \log _{2}\left(1+\frac{\tilde{p}_{\ell k}\left|h_{\ell k}\right|^{2}}{W N_{0}}\right), \\
\forall \ell \in \mathcal{L}, \forall k \in \mathcal{K} ., \forall d \in \mathcal{D},
\end{gathered}
$$

Since this problem uses potentially suboptimal power allocations, the weighted-sum rates it generates represent a lower bound on those generated by the original problem in (19).

\section{Complexity Analysis}

In this section we will examine the computational complexity required for solving the problems in (12) and (13) for the cases of continuous and binary scheduling, respectively, as well as the computational complexity required for computing the lower bounds in Sections V-A and V-B.

The formulations for the cross-layer designs provided in Sections IV and V are convex and hence highly-efficient interior-point methods (IPMs) can be utilized to attain their global optimal. The philosophy that underlies IPMs is to use the objective and the inequality constraints to synthesize a log-barrier function, which is then minimized using Newton's method along a central path. The complexity of each Newton step along this path can be shown to grow with the cube of the number of constraints, and the number of Newton steps can be bounded if the log-barrier function possesses the so-called self-concordance property [25], cf. Appendix C.

Unfortunately, the log-barrier functions corresponding to the formulations in (12), (15) and (23) are not self-concordant. Hence, to assess the complexity of solving them, in Appendix $\mathrm{C}$ we introduce auxiliary constraints that do not affect the solution, but enables us to cast the log-barrier function in a self-concordant form. Using this approach we arrive at the following results.

Proposition 1. The complexity of solving (12), (15) and (23) is of order $\mathcal{O}\left(m^{3.5}\right)$, where

- for the optimal JRSPA with time-sharing (Continuous Scheduling),

$$
m=L K(4+D)+N+K+D(N-1) ;
$$


- for the lower bound on binary JRSPA (Rounding-Based Approach),

$$
m=L K(3+D)+N+D(N-1) ; \quad \text { and }
$$

- for the lower bound on binary JRSPA (GP-Based Approach),

$$
m=L K(3+D+(L-1) / 2)+N+D(N-1) .
$$

Using exhaustive search over the binary schedules, the complexity of solving the optimal JRSPA without time-sharing (cf. (13)) is bounded by

$$
\mathcal{O}\left((L+1)^{K}(L K(3+D)+N+D(N-1))^{3.5}\right) .
$$

Proof: See Appendix C.

Note that the GP-based lower bound is obtained by solving a sequence of GPs of the form in (23) iteratively; the computational complexity of each iteration is provided in the third statement of Proposition 1. Simulation results provided herein and in [4], [26]-[29] suggest that the GP-based approach converges within 100 iterations, irrespective of the number of nodes, $N$.

Proposition 1 shows that the computational complexity required for finding the optimal JRSPA design with timesharing and the lower bounds on the optimal JRSPA without time-sharing are polynomial in the number of nodes, $N$, and subchannels, $K$. Hence, the complexities of the optimal design and the lower bounds are relatively small and comparable. In particular, the computational complexity of the optimal JRSPA with time-sharing and the rounding-based lower bound on the optimal JRSPA without time-sharing grows as $K^{3.5} N^{7}$, whereas the corresponding complexity for the GP-based lower bound grows as $K^{3.5} N^{14}$. In contrast, the computational complexity required for finding the optimal JRSPA without time-sharing is polynomial in $N$, but exponential in $K$.

\section{NUMERICAL RESULTS}

In this section we provide numerical examples to evaluate the performance of the cross-layer design approaches presented in Sections IV and V for both continuous and binary scheduling frameworks. We present four examples. In the first example, we assess the performance of the optimal JRSPA design with time-sharing and compare it with its binary rounding-based counterpart. In the second example, we apply the design techniques of Sections IV and V in a 10node LTE-based network. We illustrate the sum rate achieved by these techniques and the corresponding routes, schedules and power allocations. In the third example, we consider the case of binary scheduling in a 4-node network. We compare the rounding-based and the GP-based lower bounds with the upper bound, corresponding to continuous scheduling, and the optimal binary schedules, obtained by exhaustive search. In the last example, the region of rates achievable by the design techniques of Sections IV and V is plotted for an exemplary 4-node network. For all examples, the mathematical programs are solved using the CVX package [30] with underlying Sedumi [31] and MOSEK [32] solvers.

The nodes of the wireless networks considered in this section are randomly dropped on a $100 \times 100 \mathrm{~m}^{2}$ square and assumed to have identical power budgets, $P_{n}=P, n=$
$1, \cdots, N$. In accordance with the IMT-Advanced scenario [21], the available bandwidth, $W_{0}$, is set to $20 \mathrm{MHz}$ and the thermal noise power density is set to $-174 \mathrm{dBm} / \mathrm{Hz}$. The subchannels are assumed to be standard quasi-static frequencyflat Rayleigh fading with log-normal shadowing and pathloss components. The gains of these subchannels are given by

$$
\left|h_{\ell k}\right|^{2}=10^{-0.1 S_{\ell}-0.1 \rho(\ell)}\left|h_{\ell k}^{\prime}\right|^{2}, \quad \forall \ell \in \mathcal{L}, \forall k \in \mathcal{K},
$$

where $S_{\ell}$ represents the shadowing component, which depends on the propagation environment urban or otherwise. Using the non line-of-sight (NLoS) model of indoor hotspot (InH) scenario in [21], the shadowing component, $S_{\ell}$, is assumed to be Gaussian-distributed in the logarithmic domain with a mean of $0 \mathrm{~dB}$ and a standard deviation of $\sigma_{s}=4 \mathrm{~dB}$. In (26), $\rho(\ell)$ represents the pathloss component, which depends on the length of link $\ell, d_{\ell}$, i.e., the distance between nodes $n$ and $n^{\prime}$. An expression for $\rho(\ell)$ that conforms to the InH-NLoS standard model in [21] is $\rho(\ell)=43.3 \log _{10}\left(d_{\ell}\right)+11.5+$ $20 \log _{10}\left(f_{c}\right)$ where $f_{c}$ is the carrier frequency in $\mathrm{GHz}$ which is set to $f_{c}=3.4 \mathrm{GHz}$. The Rayleigh fading component in the channel model in (26) is captured by $\left|h_{\ell k}^{\prime}\right|^{2}$, where $h_{\ell k}^{\prime}$ is a zero mean unit variance complex Gaussian-distributed random variable.

In implementing the GP-based design, the value of $\epsilon$ in (23h) is set to $10^{-4}$.

Example 1: (Average Performance) In this example, we use Mont Carlo simulations to assess the average performance of the optimal JRSPA design with time-sharing in Section IV and compare it with its binary rounding-based counterpart in Section V-A and a heuristic algorithm in which the formulation in (10) is solved with power being equally allocated to all subchannels. Simulation results are averaged over 100 independent realizations of a network with $N=10$ nodes. Among these nodes, five are set to be source-destination nodes, i.e., $|\mathcal{D}|=5$, and the remaining five nodes can only act as relays. All weights are set equal to one. The number of directional links is $L=90$, and the number of subchannels available for all links is $K=4$.

The sum rates yielded by the optimal JRSPA design with time-sharing, the rounding-based design and the heuristic algorithm are depicted in Figure 2 for $P$ ranging from 0 to $30 \mathrm{dBm}$. From this figure, it can be seen that, both the continuous and binary designs proposed in Sections IV and V-A, outperform the heuristic algorithm and as expected, the optimal JRSPA design with time-sharing yields better performance than its binary rounding-based counterpart. However, the performance difference between these designs is relatively small. This suggests that the average performance of the rounding-based approach can be close to the optimum binary one, but requires a significantly less computational effort.

Example 2: (Performance Evaluation) To illustrate the routes, schedules and power allocations generated by the techniques of Sections IV and V, we consider an exemplary network of $N=10$ nodes. Among these nodes, three are set to be source-destination nodes, i.e., $\mathcal{D}=\{1,2,3\}$, and the remaining seven nodes can only act as relays. To reduce the number of optimization variables and avoid over-complicating the design, we ignore the link between any two nodes with distance greater than a threshold, $d_{T}$. For this example, $d_{T}$ is 


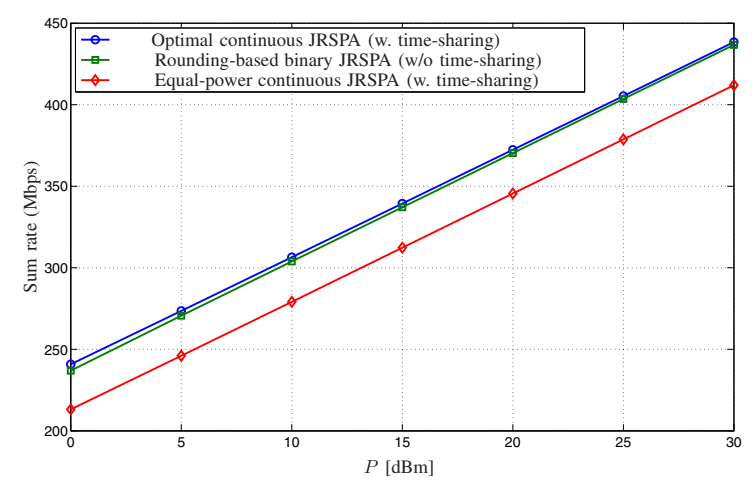

Fig. 2: (Example 1) Sum rate generated by optimal, heuristic and rounding-based JRSPA designs.

set to be half the square root of the total area, that is, for the considered example $d_{T}=50$. This setting results in reducing the number of directional links available for communication from $L=90$ to $L=42$. The number of subchannels is assumed to be $K=8$. Providing all subchannel gains of the considered network is not possible due to space limitations, but since these gains are dominated by the pathloss component, in Figure 3(a) we provide the geographic location of the nodes. In this figure, the source-destination nodes are the numbered circles, and the relay nodes are the black circles.

The cross-layer design corresponding to the network under consideration has 1707 variables of which, 336 are power variables, 336 are scheduling variables and 1035 are routing variables.

Our objective in this example is to maximize the sum rate, i.e., we set $w_{n}^{(d)}=1$, for $n, d \in\{1,2,3\}, n \neq d$. Setting $P=25 \mathrm{dBm}$, the optimal JRSPA design in (12) yields a sum rate of 168 megabits per second (Mbps), the rounding-based approach in (15) yields a sum rate of $130 \mathrm{Mbps}$, and the GPbased approach in (23) yields a sum rate of $123.3 \mathrm{Mbps}$. The sequential algorithm used to obtain the GP-based lower bound is initiated at the power allocation of $\sqrt{\epsilon}$ for all $\ell \in \mathcal{L}, k \in \mathcal{K}$ and converges within 12 iterations.

In Figures 3(b), 3(c) and 3(d), we illustrate the routes yielded by the optimal design with time-sharing, the roundingbased binary design and the GP-based binary design, respectively. The thickness of any of the links depicted in these figures is chosen to be proportional to the aggregate data rate communicated over that link. Although the sum rates yielded by these designs, are relatively close to each other, the structure of their routing, scheduling and power allocations are different. Therefore, it is fathomable that, for some scenarios, one of these designs may be preferred over other ones due to its specific structure.

In Figure 4, we provide a comparison between the sum rates yielded by the optimal JRSPA design with time-sharing, the rounding-based design, and the GP-based design for various node power budgets. From this figure, it can be seen that, for the considered network, the performance of the GP-based approach is better than that of the rounding-based one for values of $P$ lower than $22 \mathrm{dBm}$ and for values of $P$ higher than $22 \mathrm{dBm}$, the performance of the rounding-based approach is better than that of the GP-based one.

Example 3: (Comparison with the Optimal Binary Solution)

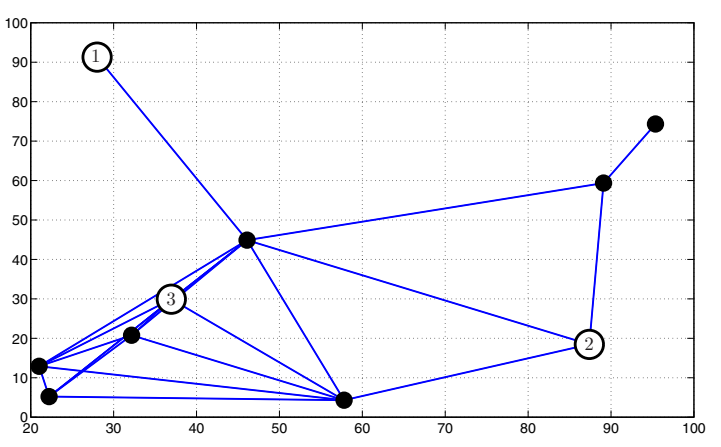

(a) Network topology

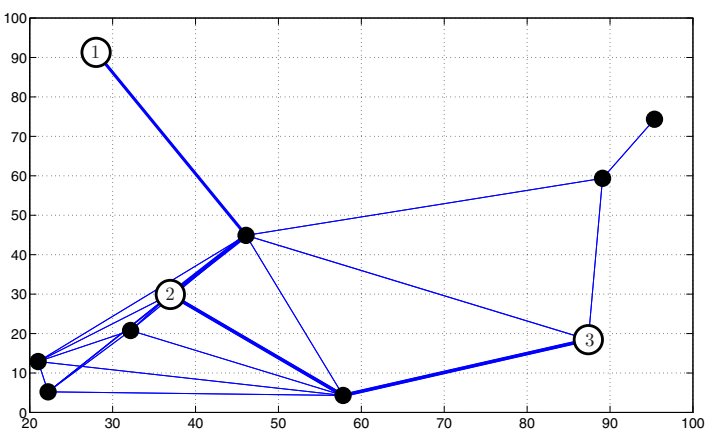

(b) Routing-Optimal continuous (w. time-sharing)

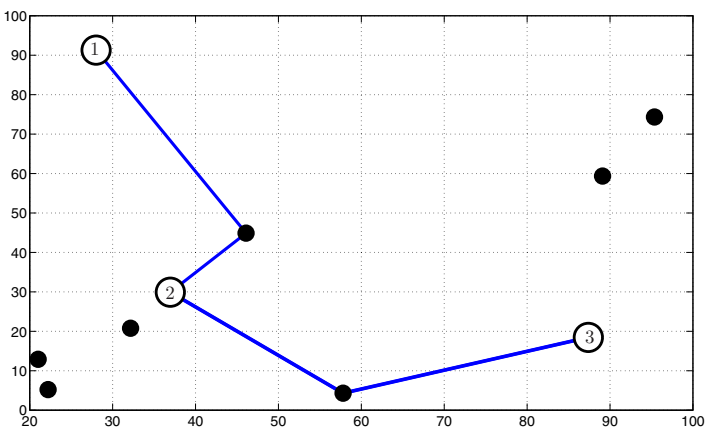

(c) Routing-Rounding-based binary (w/o time-sharing)

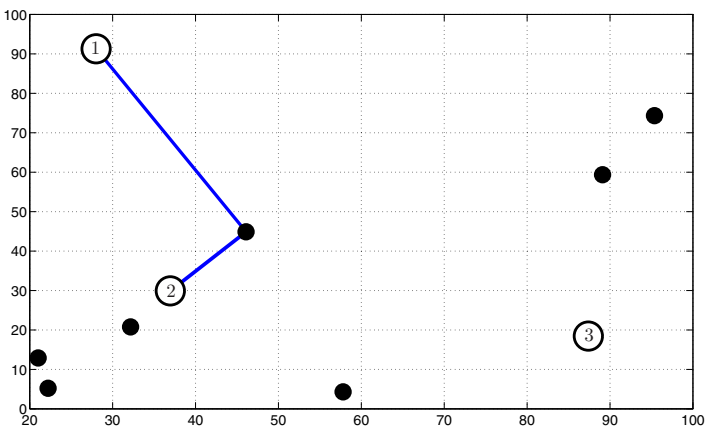

(d) Routing-GP-based binary (w/o time-sharing)

Fig. 3: Routing by different designs for the 10-node network in Example 2. 


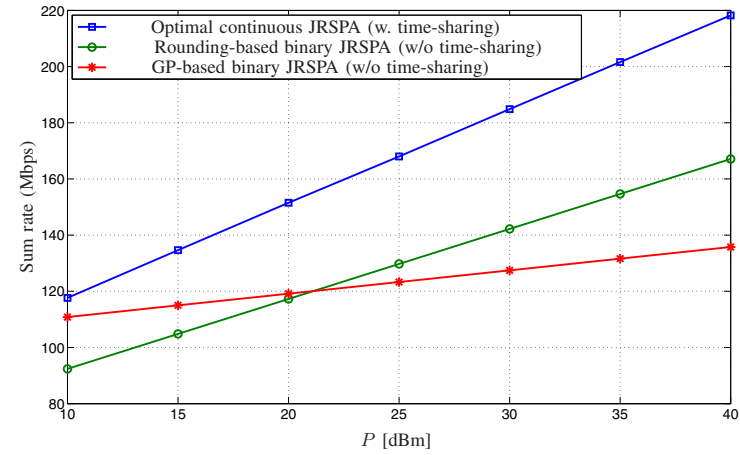

Fig. 4: (Example 2) Sum rate generated by optimal, roundingbased and GP-based JRSPA.

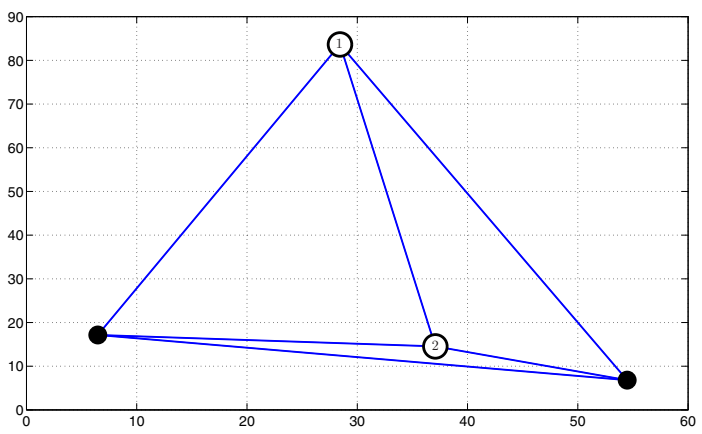

Fig. 5: Topology of the network considered in Example 3.

Since exhaustive search is computationally infeasible for large networks, to illustrate the relative tightness of the proposed bounds, in this example we compare our results with the optimal binary solution obtained through exhaustive search for a small network with $N=4$ nodes; cf. Figure 5. In this network, two nodes are set to be source-destination nodes, i.e., $D=\{1,2\}$ and the remaining two nodes are restricted to be relays only. The number of links and subchannels are $L=12$ and $K=2$.

We compare the upper and lower bounds yielded by the formulations in Sections IV and V, respectively, with the true maximum sum rate obtained by exhaustive search over all possible binary schedules, i.e., the sum rate that maximizes (13). This sum rate is obtained by solving a convex optimization problem analogous to the one in (15) for each feasible (binary) schedule. The optimal binary schedule is the one which yields the highest sum rate.

Our objective in this example is to maximize the sum rate, i.e., we set $w_{n}^{(d)}=1$, for $n, d \in\{1,2\}, n \neq d$. The rates achieved by the aforementioned schemes are plotted in Figure 6 for $P$ ranging from 10 to $40 \mathrm{dBm}$. This simulation suggests that, at low values of $P$, the GP-based approach provides a higher sum rate than the rounding-based one. However, at high values of $P$, the rounding-based approach yields a higher sum rate than the GP-based one. This is because, at low values of $P$, there are fewer good-quality links and consequently fewer route alternatives. In that case, the optimal signalling strategy is likely to rely on routes with subchannel sharing. On the other hand, at high values of $P$, there are more route alternatives and the chances of having a better performance without sharing subchannels is higher. Hence, in that region, the performance of the rounding-based approach is likely to be superior to the performance of the

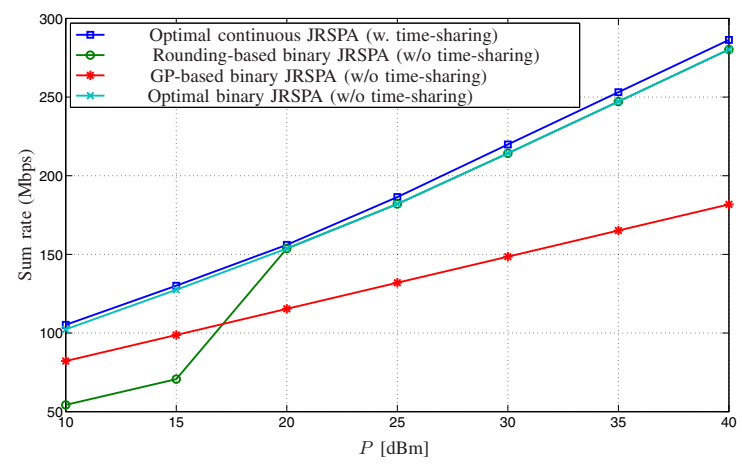

Fig. 6: (Example 3) Sum rate generated by upper and lower bounds and optimal binary design.

GP-based one. This comes in agreement with the behaviour of the network considered in the previous example, cf. Figure 4. Extensive numerical experiments suggest that these behaviours are intimately related to channel gains and network topologies. To elaborate on this observation, we note that, for the GPbased approach, the underlying iterative monomial approximation technique is known to yield a solution that satisfies the KKT conditions. This implies that this approach would yield the optimal design had the initial point been appropriately chosen. Unfortunately, finding such an initial point depends on the network structure and is generally elusive. In contrast, the rounding-based approach does not depend on an initial point. This is because this approach depends on rounding the solution of a strictly convex problem. In other words, the roundingbased approach depends solely on the network structure.

Example 4: (Achievable Rate Region) In this example, we provide the rate regions that can be achieved by the design techniques in Sections IV and V for a network with $N=4$ nodes and $K=2$ subchannels in which two nodes are sourcedestination nodes, i.e., $D=\{1,2\}$ and the remaining two nodes act as relays only. The channel gains in this example are

$$
\begin{aligned}
& {\left[\left|h_{\ell k}\right|^{2}\right]^{T}=10^{-8} \times\left[\begin{array}{llllll}
.004 & .118 & .001 & .001 & .251 & 2.69 \\
.012 & .01 & .001 & .017 & .01 & 1.79
\end{array}\right.} \\
& \left.\begin{array}{llllll}
0 & .37 & 0 & .001 & .39 & .057 \\
.006 & .341 & .006 & 0 & .864 & .032
\end{array}\right] .
\end{aligned}
$$

Applying the cross-layer design techniques in Sections IV and $\mathrm{V}$ for all $w_{2}^{(1)}$ and $w_{1}^{(2)}$ in the unit simplex $\left\{\left(w_{2}^{(1)}, w_{1}^{(2)}\right) \mid w_{2}^{(1)} \geq 0, w_{1}^{(2)} \geq 0, w_{2}^{(1)}+w_{1}^{(2)}=1\right\}$, we obtain the region of all rate tuples $\left(s_{2}^{(1)}, s_{1}^{(2)}\right)$ that can be achieved over the considered network. For $P=20 \mathrm{dBm}$, these regions are shown in Figure 7. Since the optimal continuous JRSPA design is derived from a convex formulation involving the pair $\left(s_{2}^{(1)}, s_{1}^{(2)}\right)$, it is straightforward to see that the region of rate tuples $\left(s_{2}^{(1)}, s_{1}^{(2)}\right)$ generated by this design is also convex for any given network. It can be seen from the figure that the optimal continuous JRSPA design yields a greater rate region than the GP-based and the rounding-based approaches. Also it can be seen that, in comparison with the roundingbased approach, the GP-based approach yields larger values of $s_{1}^{(2)}$ for some values of $s_{2}^{(1)}$, whereas, for other values of $s_{2}^{(1)}$, it yields smaller values of $s_{1}^{(2)}$. 


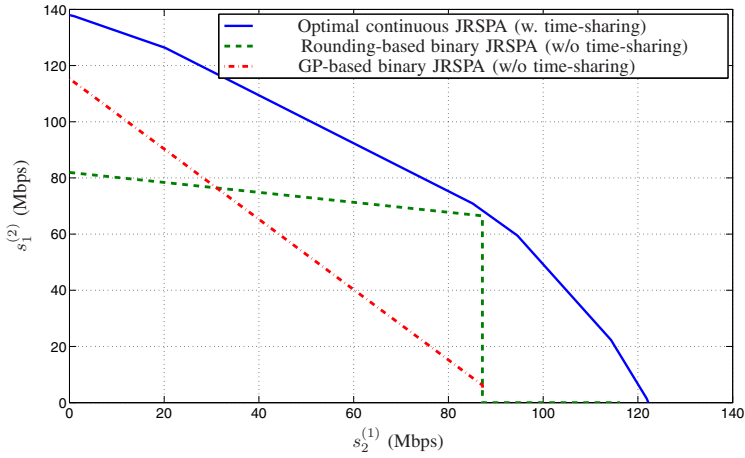

Fig. 7: Achievable rate region for the network in Example 4 with the channel gains in (27).

\section{CONCLUSION}

In this paper we considered the joint optimization of data routes, subchannel schedules and power allocations in a generic OFDMA-based wireless network with an ad hoc topology. Two instances of this problem are considered. In the first instance, subchannels are allowed to be time-shared by multiple links, whereas in the second instance, each subchannel is exclusively used by one of the links throughout the signalling duration. The first problem is transformed into an efficiently solvable convex form. In contrast, the second problem is not amenable to such a transformation and results in a complex mixed integer optimization problem that is difficult to solve. To alleviate this difficulty, we obtained efficiently computable upper and lower bounds on the weighted-sum rate that can be reliably communicated over such a network. Numerical investigations show that the obtained rates are higher when time-sharing is allowed, and that the lower bounds on rates in the absence of time-sharing are relatively tight.

\section{APPENDIX A \\ THE GP STANDARD FORM AND MONOMIAL APPROXIMATION}

1) The GP Standard Form: A GP optimization problem can be readily transformed to an efficiently solvable convex one. To provide the standard form of a GP, let $z \in \mathbb{R}^{n}$ be a vector of positive entries. A monomial in $z$ is defined to be a function of the form $c_{0} \prod_{i} z_{i}^{\alpha_{i}}$ and a posynomial in $z$ is defined to be a function of the form $\sum_{k} c_{k} \prod_{i} z_{i}^{\alpha_{i k}}$, where $c_{k}>0,\left\{\alpha_{i}\right\}$ and $\left\{\alpha_{i k}\right\}$, are arbitrary constants, $k=0,1, \ldots$, and $i=1, \ldots, n$. A standard GP is an optimization problem of the following form with $\left\{f_{i}\right\}$ being posynomials and $\left\{g_{i}\right\}$ being monomials [4], [22]:

$$
\begin{aligned}
& \min _{z} f_{0}(z), \\
& \text { subject to } f_{i}(z) \leq 1 \text {, } \\
& i=1, \ldots, m \text {, } \\
& g_{i}(z)=1 \text {, } \\
& i=1, \ldots, p \text {. }
\end{aligned}
$$

2) Monomial Approximation: A monomial approximation of a differentiable function $h(z) \geq 0$ near $z^{(0)}$ is given by its first order Taylor expansion in the logarithmic domain [22]. Defining $\beta_{i}=\left.\frac{z_{i}^{(0)}}{h\left(z^{(0)}\right)} \frac{\partial h}{\partial z_{i}}\right|_{z=z^{(0)}}$, we have

$$
h(z) \approx h\left(z^{(0)}\right) \prod_{i=1}^{n}\left(\frac{z_{i}}{z_{i}^{(0)}}\right)^{\beta_{i}} .
$$

This approximation is used to provide a GP approximation of the cross-layer design problem.

\section{APPENDIX B \\ Convergence of the Single Condensation Method IN SECTION V-B}

In the GP-based approach in Section V-B, the technique in Appendix A-2 is used to approximate each posynomial on the RHS of (22) by a monomial. The solution of the GP resulting from this approximation is then used as an initial point for the next iteration and so on. The iterates generated by this sequential algorithm converge to a solution of the KKT system corresponding to the problem in (19) if the conditions outlined in [4] and [33] are satisfied. For the affine posynomials on the RHS of (22), these conditions can be expressed as

$$
\begin{aligned}
& \text { 1) } q_{\ell k}\left(p_{\ell k} / p_{\ell k}^{(0)}\right)^{\theta_{\ell k}} \leq W N_{0}+p_{\ell k}\left|h_{\ell k}\right|^{2}, \\
& \text { 2) }\left.q_{\ell k}\left(p_{\ell k} / p_{\ell k}^{(0)}\right)^{\theta_{\ell k}}\right|_{p_{\ell k}=p_{\ell k}^{(0)}}=W N_{0}+\left.p_{\ell k}\left|h_{\ell k}\right|^{2}\right|_{p_{\ell k}=p_{\ell k}^{(0)}}, \\
& \text { 3) }\left.\frac{\partial\left(q_{\ell k}^{-1}\left(p_{\ell k}^{(0)} / p_{\ell k}\right)^{\left.\theta_{\ell k}\right)}\right.}{\partial p_{\ell k}}\right|_{p_{\ell k}=p_{\ell k}^{*}}=\left.\frac{\partial\left(W N_{0}+p_{\ell k}\left|h_{\ell k}\right|^{2}\right)^{-1}}{\partial p_{\ell k}}\right|_{p_{\ell k}=p_{\ell k}^{*}},
\end{aligned}
$$

where at each iteration $q_{\ell k}=W N_{0}+p_{\ell k}^{(0)}\left|h_{\ell k}\right|^{2}, \theta_{\ell k}=$ $\frac{p_{\ell k}^{(0)}\left|h_{\ell k}\right|^{2}}{q_{\ell k}}$ and $p_{\ell k}^{*}$ is the power allocation at convergence. These conditions are known to be satisfied by affine functions (see e.g., [15]), which guarantees convergence of the single condensation method.

\section{APPENDIX C \\ Proof OF Proposition 1}

The proof of Proposition 1 hinges on the assumption that the log-barrier functions of the problems considered therein possess the self-concordant property which is defined as follows:

Definition 1. A function $f: \mathbb{R}^{n} \rightarrow \mathbb{R}$ is said to be selfconcordant if, for all $x, v \in \mathbb{R}^{n}, s \in \mathbb{R}$ such that $x+s v$ is in the domain of $f$ and $\left|\frac{\partial^{3}}{\partial s^{3}} f(x+s v)\right| \leq 2 \frac{\partial^{2}}{\partial s^{2}} f(x+s v)^{3 / 2}$.

\section{A. Proving the First Statement of Proposition 1}

To bound the complexity of solving the problem (12), we begin by noting that its log-barrier function is not selfconcordant. This difficulty can be circumvented by adding the following auxiliary set of constraints which have no effect on the actual feasible set or the final solution:

$$
c_{\ell k}+\frac{y_{\ell k}\left|h_{\ell k}\right|^{2}}{W N_{0}} \geq 0, \quad \forall k \in \mathcal{K}, \ell \in \mathcal{L} .
$$

To construct the log-barrier function, $\psi$, the sum of the logarithm of the inequality constraints in (12) and the auxiliary constraints in (30) is superimposed on the scaled objective in (12). In particular, using $t$ to denote the non-negative scalar that multiplies the objective of the log-barrier function of the IPM, we can write

$$
\begin{gathered}
\psi=\phi-\sum_{\ell, k} \log \left(c_{\ell k} \log \left(1+\frac{y_{\ell k}\left|h_{\ell k}\right|^{2}}{W N_{0} c_{\ell k}}\right)-\sum_{d} \frac{x_{\ell k}^{(d)}}{W}\right) \\
-\sum_{\ell, k} \log \left(c_{\ell k}+\frac{y_{\ell k}\left|h_{\ell k}\right|^{2}}{W N_{0}}\right),
\end{gathered}
$$




$$
\begin{aligned}
\phi= & -t \sum_{n} \sum_{d} w_{n}^{(d)} s_{n}^{(d)}-\sum_{\ell, k, d} \log \left(x_{\ell k}^{(d)}\right)-\sum_{n, d} \log \left(s_{n}^{(d)}\right) \\
& -\sum_{\ell, k} \log \left(c_{\ell k}\right)-\sum_{\ell, k} \log \left(y_{\ell k}\right)-\sum_{k} \log \left(1-\sum_{\ell} c_{\ell k}\right) \\
& -\sum_{n} \log \left(P_{n}-\sum_{\ell, k} y_{\ell k}\right) .
\end{aligned}
$$

The function $\phi$ represents the log-barrier function of a linear optimization problem, which is known to be selfconcordant [24]. It remains to show the self-concordance of the last two terms of (31). To show this, we add and subtract $2 \sum_{\ell, k} \log c_{\ell k}$ to (31). Hence,

$$
\begin{aligned}
\psi=\phi-2 \log \left(c_{\ell k}\right)-\sum_{\ell, k} \log ( & \left.\log \left(1+\frac{y_{\ell k}\left|h_{\ell k}\right|^{2}}{W N_{0} c_{\ell k}}\right)-\frac{\sum_{d} x_{\ell k}^{(d)}}{W c_{\ell k}}\right) \\
& -\log \left(1+\frac{y_{\ell k}\left|h_{\ell k}\right|^{2}}{W N_{0} c_{\ell k}}\right) .
\end{aligned}
$$

Now, the first two terms of (32) are self-concordant. To show the self-concordance of the last two terms, we note that these terms can be expressed in the form $-\log b-\log (\log b-a)$, where $a=\frac{\sum_{d} x_{\ell k}^{(d)}}{W c_{\ell k}}$ and $b=1+\frac{y_{\ell k}\left|h_{\ell k}^{2}\right|}{W N_{0} c_{\ell k}}$. This form is known to be self-concordant [24], which establishes the fact that the log-barrier function corresponding to the problem (12) is self-concordant. We will now use this result to bound the complexity of the IPM that solves (12).

With the log-barrier function possessing the selfconcordance property, the number of Newton iterations required to obtain the solution of (12) can be shown to be proportional to $\sqrt{m}$ where $m$ is the number of inequality constraints. In addition, each Newton step is known to have a cubic complexity [34]. Hence the computational complexity of finding the optimal continuous scheduling-based design in (12) can be bounded by $\mathcal{O}\left((L K(4+D)+N+K+D(N-1))^{3.5}\right)$, which completes the proof of the first statement of Proposition 1.

\section{B. Proving the Second Statement of Proposition 1}

To prove the second statement, we note that the roundingbased lower bound requires solving (12) followed by solving (15) with the corresponding fixed rounded schedules.

To bound the complexity of solving (15), we show that the log-barrier function corresponding to this problem is selfconcordant. To do so, we add the following auxiliary set of constraints:

$$
1+\frac{p_{\ell k}\left|h_{\ell k}^{2}\right|}{W N_{0}} \geq 0, \quad \forall \ell \in \mathcal{L}, k \in \mathcal{K} .
$$

Invoking these constraints and arguing along the same lines as in the proof of the first statement, it can be shown that the capacity constraints in (15e) can be cast in the self-concordant form. Using this result the complexity of solving (15) with IPM can be shown to be bounded by $\mathcal{O}((L K(3+D)+N+$ $\left.D(N-1))^{3.5}\right)$. Details are omitted for brevity.

\section{Proving the Third Statement of Proposition 1}

Each iteration of the the GP-based approach involves solving a GP of the form in (23). Such a GP is converted to a convex problem using a standard exponential change of variables. Using auxiliary variables to bound the exponentially transformed variables in $(23 \mathrm{~g})$, the $\log$-barrier function corresponding to (23) can be shown to be self-concordant [24]. Using this result, the complexity of solving the GP in (23) can be shown to be bounded by $\mathcal{O}((L K(3+D+(L-1) / 2)+$ $\left.N+D(N-1))^{3.5}\right)$. See [24] for more details.

\section{Proving the Last Statement of Proposition 1}

The optimal solution of (13) can be found by using exhaustive search over all possible binary schedules. Each feasible schedule, satisfying (10h), corresponds to a situation in which each subchannel is either not used by any of the $L$ links or used by one of them. Since there are $K$ subchannels, the number of possible schedules to search over is $(L+1)^{K}$. For each schedule, a convex optimization problem similar to the one in (15) is solved using IPM, which implies that the overall complexity of solving the JRSPA problem in (13) is bounded by $\mathcal{O}\left((L+1)^{K}(L K(3+D)+N+D(N-1))^{3.5}\right)$. This completes the proof of the last statement of Proposition 1.

\section{ACKNOWLEDGMENT}

The authors would like to thank Dr. Chandra (Sekhar) Bontu and Dr. Jim Womack of BlackBerry (formerly Research In Motion (RIM)) for their support.

\section{REFERENCES}

[1] H. Yanikomeroglu, "Fixed and mobile relaying technologies for cellular networks," in Proc. 2002 Wksp. Apps Svcs. Wireless Netw., pp. 75-81.

[2] J. Leonard and J. Cimini, "Analysis and simulation of a digital mobile channel using orthogonal frequency division multiplexing," IEEE Trans. Commun., vol. 33, pp. 665-675, July 1985.

[3] S. Sadr, A. Anpalagan, and K. Raahemifar, "Radio resource allocation algorithms for the downlink of multiuser OFDM communication systems," IEEE Commun. Surv. Tutorial, vol. 11, pp. 92-106, Aug. 2009.

[4] M. Chiang, C. W. Tan, D. P. Palomar, D. O'Neil, and D. Julian, "Power control by geometric programming," IEEE Trans. Wireless Commun., vol. 6, pp. 2640-2650, July 2007.

[5] L. Liu, R. Zhang, and K.-C. Chua, "Achieving global optimality for weighted sum-rate maximization in the K-user Gaussian interference channel with multiple antennas," IEEE Trans. Wireless Commun., vol. 11, pp. 2640-2650, May 2012.

[6] H. Inaltekin and S. V. Hanly, "Optimality of binary power control for the single cell uplink," IEEE Trans. Inf. Theory, vol. 58, pp. 6484-6498, Oct. 2012.

[7] J. Jang and K. B. Lee, "Transmit power adaptation for multiuser OFDM systems," IEEE J. Sel. Areas Commun., vol. 21, pp. 171-178, Feb. 2003.

[8] G. Song and Y. Li, "Utility-based joint physical-MAC layer optimization in OFDM," in Proc. 2002 IEEE Glob. Commun. Conf., vol. 1, pp. 671675.

[9] C. Y. Wong, R. S. Cheng, K. B. Lataief, and R. D. Murch, "Multiuser OFDM with adaptive subcarrier, bit, and power allocation," IEEE J. Sel. Areas Commun., vol. 17, no. 10, pp. 1747-1758, Oct. 1999.

[10] J. Huang, V. G. Subramanian, R. Agrawal, and R. A. Berry, "Downlink scheduling and resource allocation for OFDM systems," IEEE Trans. Wireless Commun., vol. 8, pp. 288-296, Jan. 2009.

[11] K. Kim, Y. Han, and S.-L. Kim, "Joint subcarrier and power allocation in uplink OFDMA systems," IEEE Commun. Lett., vol. 9, pp. 526-528, June 2005.

[12] C. Y. Ng and C. W. Sung, "Low complexity subcarrier and power allocation for utility maximization in uplink OFDMA systems," IEEE Trans. Wireless Commun., vol. 7, pp. 1667-1675, May 2008.

[13] L. Xiao, M. Johansson, and S. P. Boyd, "Simultaneous routing and resource allocation via dual decomposition," IEEE Trans. Commun., vol. 52, pp. 1136-1144, July 2004.

[14] M. Johansson and L. Xiao, "Cross-layer optimization of wireless networks using nonlinear column generation," IEEE Trans. Wireless Commun., vol. 5, pp. 435-445, Feb. 2006. 
[15] R. H. Gohary and T. J. Willink, "Joint routing and resource allocation via superposition coding for wireless data networks," IEEE Trans. Signal Process., vol. 58, pp. 6387-6399, Dec. 2010.

[16] S. Shabdanov, P. Mitran, and C. Rosenberg, "Cross-layer optimization using advanced physical layer techniques in wireless mesh networks," IEEE Trans. Wireless Commun., vol. 11, pp. 1622-1631, Apr. 2012.

[17] K. Karakayali, J. Kang, M. Kodialam, and K. Balachandran, "Crosslayer optimization for OFDMA-based wireless mesh backhaul networks," in Proc. 2007 IEEE Wireless Commun. Netw. Conf., pp. 276281.

[18] X. Lin, N. B. Shroff, and R. Srikant, "A tutorial on cross-layer optimization in wireless networks," IEEE J. Sel. Areas Commun., vol. 24, pp. 1452-1463, Aug. 2006.

[19] C. W. Tan, S. Friedland, and S. Low, "Nonnegative matrix inequalities and their application to nonconvex power control optimization," SIAM J. Matrix Anal. Appl., vol. 32, no. 3, pp. 1030-1055, 2011.

[20] C. W. Tan, S. Friedland, and S. H. Low, "Spectrum management in multiuser cognitive wireless networks: optimality and algorithm," IEEE J. Sel. Areas Commun., vol. 29, pp. 421-430, Feb. 2011.

[21] Int. Telecommun. Union (ITU), Guidelines for Evaluation of Radio Interface Technologies for IMT-Advanced. ITU-R: TR M.21351, Dec. 2009. Available at: http://www.itu.int/pub/R-REP-M.2135-1-2009.

[22] S. Boyd, S.-J. Kim, L. Vandenberghe, and A. Hassibi, "A tutorial on geometric programming," Optimization Eng., vol. 8, pp. 67-127, Mar. 2007.

[23] G. Li and H. Liu, "Resource allocation for OFDMA relay networks with fairness constraints," IEEE J. Sel. Areas Commun., vol. 24, pp. 20612069, Nov. 2006.

[24] S. Boyd and L. Vandenberghe, Convex Optimization. Cambridge University Press, 2004.

[25] Y. Nesterov, A. Nemirovskii, and Y. Ye, "Interior-point polynomial algorithms in convex programming," SIAM Rev., vol. 36, no. 4, pp. 682683, 1994

[26] C. W. Tan, "Optimal power control in Rayleigh-fading heterogeneous networks," in Proc. 2011 IEEE Int. Conf. Comp. Commun., pp. 2552 2560.

[27] C. W. Tan, M. Chiang, and R. Srikant, "Maximizing sum rate and minimizing MSE on multiuser downlink: Optimality, fast algorithms and equivalence via max-min SINR," IEEE Trans. Signal Process., vol. 59, no. 12, pp. 6127-6143, Dec. 2011.

[28] M. Charafeddine and A. Paulraj, "Sequential geometric programming for $2 \times 2$ interference channel power control," in Proc. 2007 IEEE Conf. Inf. Sci. Syst., pp. 185-189.

[29] C. W. Tan, D. P. Palomar, and M. Chiang, "Solving nonconvex power control problems in wireless networks: low SIR regime and distributed algorithms," in Proc. 2005 IEEE Global Commun. Conf., vol. 6, pp. 3445-3450.

[30] M. Grant and S. Boyd, CVX: Matlab software for disciplined convex programming, version 1.21, Jan. 2011. Available at: http://cvxr.com/cvx.

[31] J. F. Sturm, "Using SeDumi 1.02, a Matlab toolbox for optimization over symmetric cones," Optim. Methods Softw., vol. 11-12, pp. 625653, 1998

[32] MOSEK Apps., The MOSEK optimization toolbox for Matlab Manual, Vesion 6.0, 2012. Available at: http://www.docs.mosek.com.

[33] B. R. Marks and G. P. Wright, "A general inner approximation algorithm for nonconvex mathematical programs," Operations Research, vol. 26, pp. 681-683, Aug. 1978.

[34] G. Strang, Introduction to Linear Algebra. Wellesley-Cambridge Press, 2009.

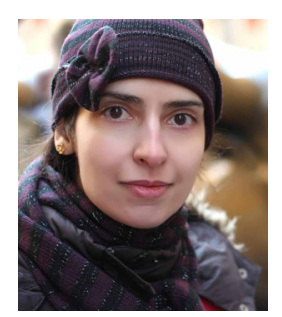

Rozita Rashtchi received the B.S. and M.Sc. degrees in Electrical Engineering from Isfahan University of Technology, Iran, in 2005 and 2008 respectively. Since 2010, she has been a Ph.D. candidate and a research assistant at the Department of Systems and Computer Engineering, Carleton University, Ottawa, Canada. During her studies toward Ph.D., she has been awarded the Carleton University President 2010 Doctoral Fellowship Award and the Graduate Scholarship Award for the academic years 2010-2014. She was a member of CarletonBlackBerry project during 2010-2012.

Her research interests include resource allocation, power control and multihop routing in wireless networks, application of optimization in wireless communication, and cross-layer design in relay-assisted networks.

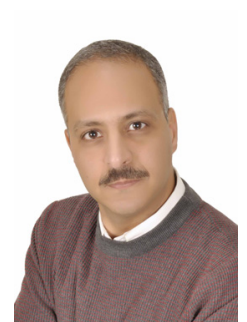

Ramy Gohary (S'02-M'06-SM'13) is an adjunct professor and a research associate with the Department of Systems and Computer Engineering, Carleton University, Ottawa, Ontario, Canada. He received the B.Eng. (Hons.) degree from Assiut University, Egypt in 1996, the M.Sc. degree from Cairo University, Egypt, in 2000, and the Ph.D. degree from McMaster University, Ontario, Canada in 2006, all in electronics and communications engineering. He received the Natural Sciences and Engineering Research Council visiting fellowship award in 2007.

From 2006 to 2007 Dr. Gohary was a postdoctoral fellow with the McMaster University. From 2008 to 2010 he was a visiting scientist with the Terrestrial Wireless Systems Branch, Communications Research Centre, Canada. He was the project manager of the Carleton-BlackBerry (formerly Research In Motion (RIM)) research project, and he is currently a member of the Carleton-Huawei research team.

His research interests include analysis and design of MIMO wireless communication systems, applications of optimization and geometry in signal processing and communications, information theoretic aspects of multiuser communication systems, and applications of iterative detection and decoding techniques in multiple antenna and multiuser systems.

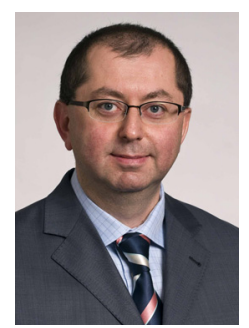

Halim Yanikomeroglu (S'96-M'98-SM'12) was born in Giresun, Turkey, in 1968. He received the B.Sc. degree in Electrical and Electronics Engineering from the Middle East Technical University, Ankara, Turkey, in 1990, and the M.A.Sc. degree in Electrical Engineering (now ECE) and the Ph.D. degree in Electrical and Computer Engineering from the University of Toronto, Canada, in 1992 and 1998, respectively. He was with the R\&D Group of Marconi Kominikasyon A.S., Ankara, Turkey, during 1993-1994.

Since 1998 Dr. Yanikomeroglu has been with the Department of Systems and Computer Engineering at Carleton University, Ottawa, where he is now a Full Professor. His research interests cover many aspects of wireless technologies with a special emphasis on cellular networks. Dr. Yanikomeroglu coauthored more than 50 IEEE journal papers, and has given a high number of tutorials and invited talks on wireless technologies in the leading international conferences. In recent years, Dr. Yanikomeroglu's research has been funded by Huawei, Blackberry, Samsung, Communications Research Centre of Canada (CRC), Telus, and Nortel. This collaborative research resulted in about 18 patents (granted and applied).

Dr. Yanikomeroglu has been involved in the organization of the IEEE Wireless Communications and Networking Conference (WCNC) from its inception, including serving as Steering Committee Member as well as the Technical Program Chair or Co-Chair of WCNC 2004, WCNC 2008, and WCNC 2014 to be held in Istanbul. Dr. Yanikomeroglu was the General Co-Chair of the IEEE Vehicular Technology Conference Fall 2010 held in Ottawa. Dr. Yanikomeroglu has served in the editorial boards of the IEEE TRANSACTIONS ON COMMUNICATIONS, IEEE TRANSACTIONS ON Wireless COMMUNiCATIONS, and IEEE CCOMMUNiCATIONS SURVEYS $\&$ Tutorials. He was the Chair of the IEEE's Technical Committee on Personal Communications (now called, Wireless Technical Committee). He is a Distinguished Lecturer for the IEEE Vehicular Technology Society.

Dr. Yanikomeroglu is a recipient of the Carleton University Faculty Graduate Mentoring Award in 2010, the Carleton University Graduate Students Association Excellence Award in Graduate Teaching in 2010, and the Carleton University Research Achievement Award in 2009. Dr. Yanikomeroglu spent the 2011-2012 academic year at TOBB University of Economics and Technology, Ankara, Turkey, as a Visiting Professor. He is a registered Professional Engineer in the province of Ontario, Canada. 